\title{
R2R3-MYB Transcription Factors Regulate Anthocyanin Biosynthesis in Grapevine Vegetative Tissues
}

\author{
Sha Xie ${ }^{1+}$, Yujuan Lei2t, Huawei Chen ${ }^{1}$, Junnan $\mathrm{Li}^{1}$, Huangzhao Chen ${ }^{1,3}$ and \\ Zhenwen Zhang ${ }^{1,4 *}$
}

${ }^{1}$ College of Enology, Northwest A\&F University, Xianyang, China, ${ }^{2}$ College of Food Science and Technology, Hebei Normal University of Science \& Technology, Qinhuangdao, China, ${ }^{3}$ College of Food and Biological Engineering, Henan University of Animal Husbandry and Economy, Zhengzhou, China, ${ }^{4}$ Shaanxi Engineering Research Center for Viti-Viniculture, Xianyang, China

\section{OPEN ACCESS}

Edited by:

Flavia Guzzo,

University of Verona, Italy

Reviewed by:

Antje Feller,

Eberhard Karls University of Tübingen,

Germany

Takashi Nakatsuka,

Shizuoka University, Japan

*Correspondence:

Zhenwen Zhang

zhangzhw60@nwsuaf.edu.cn

${ }^{\dagger}$ These authors have contributed equally to this work

Specialty section:

This article was submitted to

Plant Metabolism

and Chemodiversity,

a section of the journal

Frontiers in Plant Science

Received: 06 January 2020

Accepted: 07 April 2020

Published: 07 May 2020

Citation:

Xie S, Lei Y, Chen H, Li J, Chen H and Zhang Z (2020) R2R3-MYB

Transcription Factors Regulate Anthocyanin Biosynthesis

in Grapevine Vegetative Tissues.

Front. Plant Sci. 11:527.

doi: 10.3389/fp/s.2020.00527
Anthocyanins with important physiological functions mainly accumulate in grape berry, but teinturier grape cultivars can accumulate anthocyanins in both reproductive and vegetative tissues. The molecular regulatory mechanisms of anthocyanin biosynthesis in grapevine reproductive and vegetative tissues are different. Therefore, teinturier grapevine cultivar provides opportunities to investigate transcriptional regulation of vegetative anthocyanins, and to compare with mechanisms that regulate grape berry anthocyanins. Yan73 is a teinturier Vitis vinifera variety with vegetative tissues able to accumulate anthocyanins, but the anthocyanin pattern and the molecular mechanism regulating anthocyanin biosynthesis in these tissues remain uncharacterized. We analyzed the anthocyanin metabolic and transcriptome profiles of the vegetative tissues of Yan73 and its male parent with HPLC-ESI-MS/MS and RNA-sequencing technologies. Yan73 vegetative tissues had relatively high $3^{\prime}-\mathrm{OH}$, acylated, and methoxylated anthocyanins. Furthermore, peonidin-3-O-(trans-6-coumaryl)-glucoside is the most abundant anthocyanin in Yan73 grapevine vegetative tissues. A total of 30,17 and 10 anthocyanin biosynthesis genes showed up-regulated expression in Yan73 leaf, stem and tendril, respectively, indicating anthocyanin biosynthesis in Yan73 vegetative tissues is regulated by transcription factors. The up-regulated expression of VVMYBA1 on chromosome 2 and VVMYBA5, VVMYBA6, and VVMYBA7 on chromosome 14 are responsible for the anthocyanin patterns of Yan73 vegetative tissues. The expression of a set of R2R3-MYB C2 repressor genes is activated and may negatively regulate anthocyanin biosynthesis in Yan73 vegetative tissues. These findings enhance our understanding of anthocyanin biosynthesis in grapevine.

Keywords: anthocyanin, color pigmentation, vegetative tissues, grapevine, repressor, transcription factor

\section{INTRODUCTION}

Anthocyanins are a class of flavonoids that determine the characteristic colors of plant fruits, flowers, and vegetative tissues. They are produced in plants in response to developmental and environmental signals and perform important physiological functions related to pollination and seed distribution (Albert et al., 2015), resistance to environmental stresses (e.g., high light 
irradiance, nutrient deficiency, low temperatures, and pathogen infections), and senescence (Gutha et al., 2010). Anthocyanins in reproductive tissues (e.g., berries) attract considerable attention due to their antioxidant capacity, cardiovascular disease protectiveness and antitumoral properties (Butelli et al., 2008). However, anthocyanins produced in vegetative tissues received much less attention, although they are reported to have adaptive advantages for plants. These anthocyanins in vegetative tissues often appear transiently at specific developmental stages and may be induced by environmental factors to help plants to resist environmental stresses (Chalker-Scott, 1999; Fang et al., 2018). In most grapevine, anthocyanins mainly accumulate in reproductive tissues (e.g., berries). However, in teinturier grape cultivars, anthocyanins can accumulate in both reproductive and vegetative tissues (e.g., stems, leaves, and tendrils). Indeed, in some grape varieties, anthocyanins are usually induced in vegetative tissues in response to physiological and environmental fluctuations, such as infections by the grapevine leafroll-associated virus (GLRaV) (Gutha et al., 2010) and Botrytis cinereal (Blanco-Ulate et al., 2015), exposure to environmental stresses [e.g., UV-B radiation (Sunitha et al., 2019)], early in plant development and autumnal senescence (Matus et al., 2017). These indicate anthocyanins are not only nutritional compounds but also important for plant in the adaptation to physiological and environmental fluctuations. This may partly explain why teinturier grapevines are resistant to mildew, powdery mildew, and phylloxera (Santiago et al., 2008). Anthocyanins were high in young teinturier grapevine vegetative tissues, decreased in mature vegetative tissues, and increased in senescing vegetative tissues, indicating anthocyanins accumulated in teinturier grapevine vegetative tissues may have an important role in protecting plants against external stresses as young or senescing vegetative tissue were more susceptible to stress damage (Guan et al., 2012). Until now, investigations of teinturier grapevine varieties have mainly focused on the anthocyanin in the berry skin and flesh (Falginella et al., 2012; Xie et al., 2018, 2019). However, the vegetative anthocyanins in teinturier grapevines have not been well studied, although this investigation can advance our understanding of anthocyanin in grapevine.

Anthocyanin accumulation in plant tissues depends on the coordinated expression of anthocyanin biosynthesis genes in the flavonoid branch of the phenylpropanoid pathway. This coordinated expression is usually controlled by a ternary MYB-bHLH-WD40 (MBW) transcription complex. In this complex, the R2R3-MYB transcription factor determines the spatio-temporal patterns of anthocyanin production in plants (Albert et al., 2014; Costantini et al., 2015). The members of the R2R3-MYB gene families encode diverse domains, which result in these genes typically mediating different anthocyanin patterns (Albert et al., 2011, 2015). Recent studies revealed that anthocyanin patterns in plants are coordinately regulated by R2R3-MYB transcriptional activators and repressors, such as DPL and PHZ activators and the PhMYB27 repressor in petunia (Albert et al., 2011, 2014), the PpMYB10.1 activator and PpMYB18 repressor in peach (Zhou et al., 2019), the CsRuby1 activator and CsMYB3 repressor in citrus (Huang et al., 2019), and MYB134 and MYB115 activators as well as MYB165 and
MYB194 repressors in poplar (Ma et al., 2018). Furthermore, this transcriptional network regulating anthocyanin biosynthesis is conserved in eudicots (Albert et al., 2014). Grapevine is an important model for understanding this transcriptional regulation due to the expansion and diversification of genetic factors controlling anthocyanin biosynthesis (Matus et al., 2017). Grapevine studies have primarily focused on the MYBA1 and $M Y B A 2$ transcriptional activator genes within the berry color locus on chromosome 2 because they determine the variability of anthocyanin accumulation in berry skin (Walker et al., 2007). The recently identified MYBA5, MYBA6, and MYBA7 transcriptional activator genes within the vegetative color locus on chromosome 14 control the anthocyanin biosynthesis in grapevine vegetative tissues, indicating anthocyanin accumulation in grapevine reproductive and vegetative tissues is possibly regulated by different mechanisms (Matus et al., 2017). However, this earlier study on vegetative anthocyanin mainly focused on the nonteinturier $V$. vinifera $\mathrm{cv}$. 'Pinot Noir' young leaves and tendrils and the 'Corvina Veronese' buds. Much more research is needed to exactly understand the transcriptional regulation of vegetative anthocyanins in other grapevine varieties. Teinturier grapevine cultivar with high anthocyanin accumulated in their vegetative tissues provides opportunities to investigate transcriptional regulation of vegetative anthocyanins, and to compare with mechanisms that regulate grape berry anthocyanins. In addition, recent studies indicated that a set of R2R3-MYB C2 transcriptional repressors, including MYBC2-L1 (Cavallini et al., 2015), MYBC2-L2 (Zhu et al., 2019), MYBC2-L3 (Munoz et al., 2019) and MYB4-like (Perez-Diaz et al., 2016), negatively regulate anthocyanin biosynthesis in grapevine, suggesting the transcriptional activators and repressors may cooperatively regulate anthocyanin biosynthesis in grapevine; however, relatively little is known about the underlying mechanism. We recently revealed that the MYBA1 transcriptional activator and MYBC2-L1 repressor coordinately regulate anthocyanin biosynthesis of grape berry flesh (Xie et al., 2019), but it is unknown if a similar mechanism exists for grapevine vegetative tissue anthocyanin. In fact, the transcriptional repressors associated with anthocyanin biosynthesis in vegetative tissues have yet to be identified in grapevine.

Yan73 is a teinturier grapevine variety with anthocyanin accumulation in its vegetative tissues (e.g., leaves, stems, and tendrils) (Figure 1). The red-pigmented phenotype of Yan73 vegetative tissue is inherited from its female parent, Alicante Bouschet (Guan et al., 2012), whereas Muscat Hamburg (male parent) have green vegetative tissues. However, the anthocyanin pattern and molecular mechanism regulating anthocyanin biosynthesis in Yan73 vegetative tissues have not been elucidated. In this study, we analyzed the anthocyanin metabolic and transcriptome profiles of the leaves, stems, and tendrils of Yan73 and its male parent with HPLC-ESI-MS/MS and RNAsequencing technologies. We then screened the RNA sequencing (RNA-seq) data to identify the differentially expressed R2R3MYB family genes in the leaves, stems, and tendrils among Yan73 and its male parent. Moreover, phylogenetic and quantitative real-time (qRT)-PCR analyses were completed to identify the key regulators of anthocyanin biosynthesis in Yan73 vegetative 


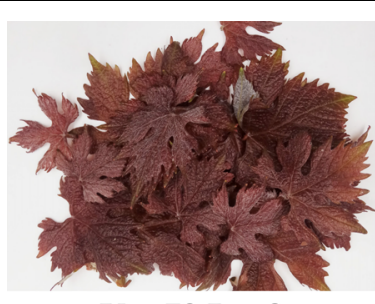

Yan73 Leaf

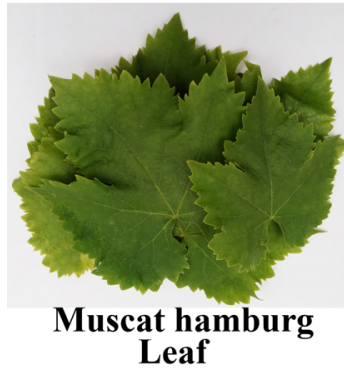

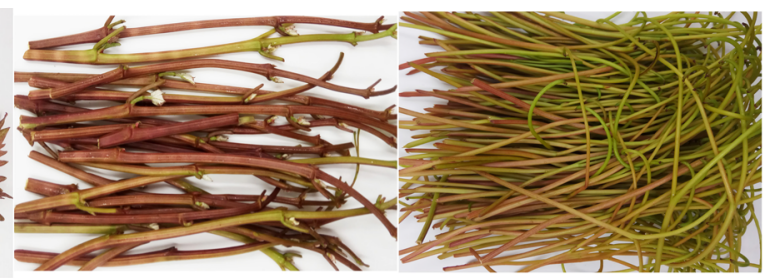

Yan73 Tendril

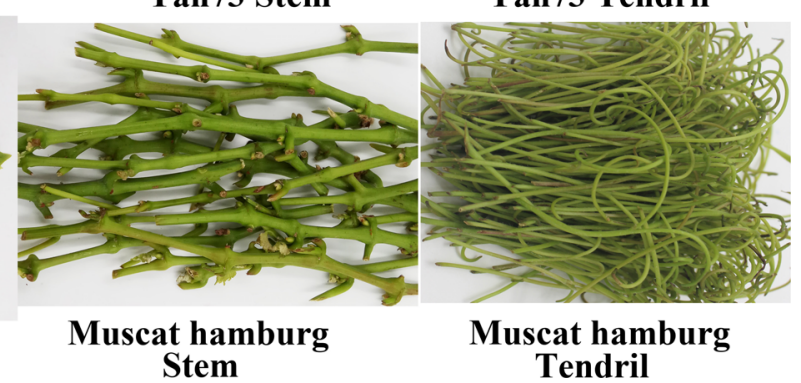

FIGURE 1 | Photos of leaf, stem, and tendril tissues from Yan73 grapevine and its male parent (Muscat Hamburg).

tissues. This study advances our understanding of anthocyanin biosynthesis in grapevine.

\section{MATERIALS AND METHODS}

\section{Plant Materials}

Leaves, stems and tendrils were collected from Vitis vinifera Yan73 and Muscat Hamburg grapevines growing at Chateau Changyu Verna in Shaanxi, China (108 73 N, $34^{\circ} 33$ E). Sample were collected following the methods described by Guan et al. (2012). Three biological replicates were collected for each tissue of each variety. When three to four new leaves emerged (01 May), 100 young fully expanded leaves of each replicate were collected from the third apical internode of grapevine shoot. At the same time as leaf sampling, the stems (nodes 1 through 3 ) and tendrils were collected. Each replicate was randomly collected from at least 60 whole grapevines on both sides of the canopy. All the samples were frozen in liquid nitrogen and stored at $-80^{\circ} \mathrm{C}$ for metabolome and transcriptome analysis.

\section{Analysis of Grapevine Vegetative Tissue Anthocyanin Compounds}

Anthocyanins were extracted from grapevine leaves, stems and tendrils and analyzed as previously described (Xie et al., 2018, 2019).

\section{RNA-Seq and Bioinformatics Analyses}

Total RNA was extracted from grapevine leaves, stems and tendrils as previously described (Yang et al., 2018; Xie et al., 2019). For each grapevine tissue, a library was constructed and sequenced using the BGISEQ-500 platform (150 bp paired ends) (Beijing Genomic Institute ${ }^{1}$ ). A transcriptome

${ }^{1}$ www.genomics.org.cn analysis was conducted by mapping the clean reads to a reference $V$. vinifera genome ${ }^{2}$. Gene expression levels were estimated using the fragments per kilobase of exon per million fragments mapped (FPKM).

\section{Identification of R2R3-MYB Family Members in Grapevine Vegetative Tissues}

Grapevine protein sequences were downloaded from a $V$. vinifera genome database $e^{2}$ to establish a local protein database. The Hidden Markov Model (HMM) profile of the R2R3-MYB domain (PF00249) was obtained from Pfam database ${ }^{3}$. This profile was used as a query to search the grapevine protein database using the HMMER program (version 3.0). Annotated protein sequences were examined for the presence of complete R2 and R3 domains with NCBI Batch Web CD-Search and HMM scan from the HMMER suite of Pfam. Finally, our RNA-seq data were selected to identify the differentially expressed R2R3-MYB family genes in the leaves, stems, and tendrils among Yan73 and its male parent.

\section{Phylogenetic Analysis}

Phylogenetic analysis was performed according to our previous report (Xie et al., 2019). Multiple sequences were aligned with the MUSCLE algorithm-based AlignX module of MEGA5.1. Phylogenetic trees were constructed using the neighbor-joining method of MEGA5.1.

\section{qRT-PCR}

A qRT-PCR assay was performed following our previous study (Xie et al., 2015). The primers used for the qRT-PCR are provided in Supplementary Table S1.

\footnotetext{
${ }^{2}$ https://www.ncbi.nlm.nih.gov/genome/?term=vitis+vinifera

${ }^{3}$ http://pfam.xfam.org/
} 


\section{Statistical Analysis}

Histograms were prepared using OriginPro (OriginLab Corporation).

\section{RESULTS}

\section{Similar Anthocyanin Profiles of Yan73 Grapevine Leaf, Stem, and Tendril \\ Tissues}

Anthocyanins in V. vinifera are mainly cyanidin-, delphinidin-, malvidin-, peonidin-, and petunidin-3-monoglucosides as well as the corresponding acetyl, $p$-coumaroyl, and caffeoyl derivatives (Guan et al., 2012; Rinaldo et al., 2015). In this study, the Yan73 grapevine red leaves contained 17 anthocyanins [four cyanidin (20.26\%), two delphinidin (11.66\%), four malvidin 8.5 (19.24\%), four peonidin (43.87\%), and three petunidin (4.97\%) derivatives], whereas the Yan73 red stems and tendrils both comprised seven anthocyanins (two cyanidin, two malvidin, and three peonidin derivatives) (Table 1). Delphinidin and petunidin derivatives were not detected in the Yan73 stems and tendrils. Additionally, the total anthocyanin concentration was more than 18-fold higher in Yan73 red leaves $(2,441.04 \mathrm{mg} / \mathrm{kg}$ dry weight) than in Yan73 red stems (134.28 mg/kg dry weight) and tendrils (132.73 mg/kg dry weight) (Table 1). Although Yan73 leaves had more anthocyanins and a higher anthocyanin concentration than Yan73 stems and tendrils, all three tissues had similar anthocyanin profiles. Specifically, peonidin derivatives accounted for more than $40 \%$ of the total anthocyanins and peonidin-3-O-(trans-6-coumaryl)-glucoside was the most abundant anthocyanin in these grapevine vegetative

TABLE 1 | Anthocyanin profiles of Yan73 grapevine leaf, stem, and tendril tissues.

\begin{tabular}{|c|c|c|c|c|}
\hline \multirow[t]{2}{*}{ Anthocyanin compounds } & \multirow[t]{2}{*}[\mathrm{M}]{$+(\mathrm{m} / \mathrm{z})$} & \multicolumn{3}{|c|}{ Concentration (mg/kg of dry weight) } \\
\hline & & Yan73 leaf & Yan73 stem & Yan73 tendril \\
\hline Cyanidin-3-O-glucoside & $449(287)$ & $166.71 \pm 0.27$ & $10.78 \pm 0.2$ & $4.74 \pm 0.18$ \\
\hline Cyanidin-3-O-(6-acetyl)-glucoside & $491(287,449)$ & $17.71 \pm 0.27$ & $N D$ & $N D$ \\
\hline Cyanidin-3-O-(6-caffeoyl)-glucoside & $611(287,449)$ & $9.44 \pm 0.22$ & ND & ND \\
\hline$\%$ & & 20.26 & 17.42 & 10.00 \\
\hline \multicolumn{5}{|l|}{ Delphinidin derivatives } \\
\hline Delphinidin-3-O-glucoside & 465(303) & $105.47 \pm 0.16$ & ND & ND \\
\hline Delphinidin-3-O-(6-coumaryl)-glucoside & $611(303,465)$ & $179.21 \pm 0.42$ & $N D$ & ND \\
\hline Subtotal & & $284.68 \pm 0.27$ & 0 & 0 \\
\hline Malvidin-3-O-(6-acetyl)-glucoside & $535(331,493)$ & $42.99 \pm 1.32$ & ND & ND \\
\hline Malvidin-3-O-(cis-6-coumaryl)-glucoside & $639(493,331)$ & $19.84 \pm 0.07$ & ND & $N D$ \\
\hline Malvidin-3-O-(trans-6-coumaryl)-glucoside & $639(493,331)$ & $267.44 \pm 0.18$ & $14.52 \pm 0.04$ & $34.14 \pm 0.40$ \\
\hline Subtotal & & $469.6 \pm 5.25$ & $30.16 \pm 0.29$ & $60.05 \pm 0.58$ \\
\hline$\%$ & & 19.24 & 22.46 & 45.24 \\
\hline \multicolumn{5}{|l|}{ Peonidin derivatives } \\
\hline Peonidin-3-O-glucoside & 463(301) & $263.33 \pm 5.02$ & $29.36 \pm 0.07$ & $18.67 \pm 0.38$ \\
\hline Peonidin-3-O-(6-caffeoyl)-glucoside & $625(463,301)$ & $42.4 \pm 0.18$ & $N D$ & $N D$ \\
\hline Peonidin-3-O-(cis-6-coumaryl)-glucoside & $609(463,301)$ & $68.52 \pm 0.22$ & $1.46 \pm 0.76$ & $0.94 \pm 0.07$ \\
\hline Peonidin-3-O-(trans-6-coumaryl)-glucoside & $609(463,301)$ & $696.72 \pm 0.81$ & $49.89 \pm 0.02$ & $39.8 \pm 0.42$ \\
\hline Subtotal & & $1070.97 \pm 5.87$ & $80.72 \pm 0.80$ & $59.41 \pm 0.74$ \\
\hline Total & & $2441.04 \pm 4.95$ & $134.28 \pm 0.91$ & $132.73 \pm 1.76$ \\
\hline
\end{tabular}

Data are presented as the mean \pm standard deviation of three biological replicates; ND, not detected. 


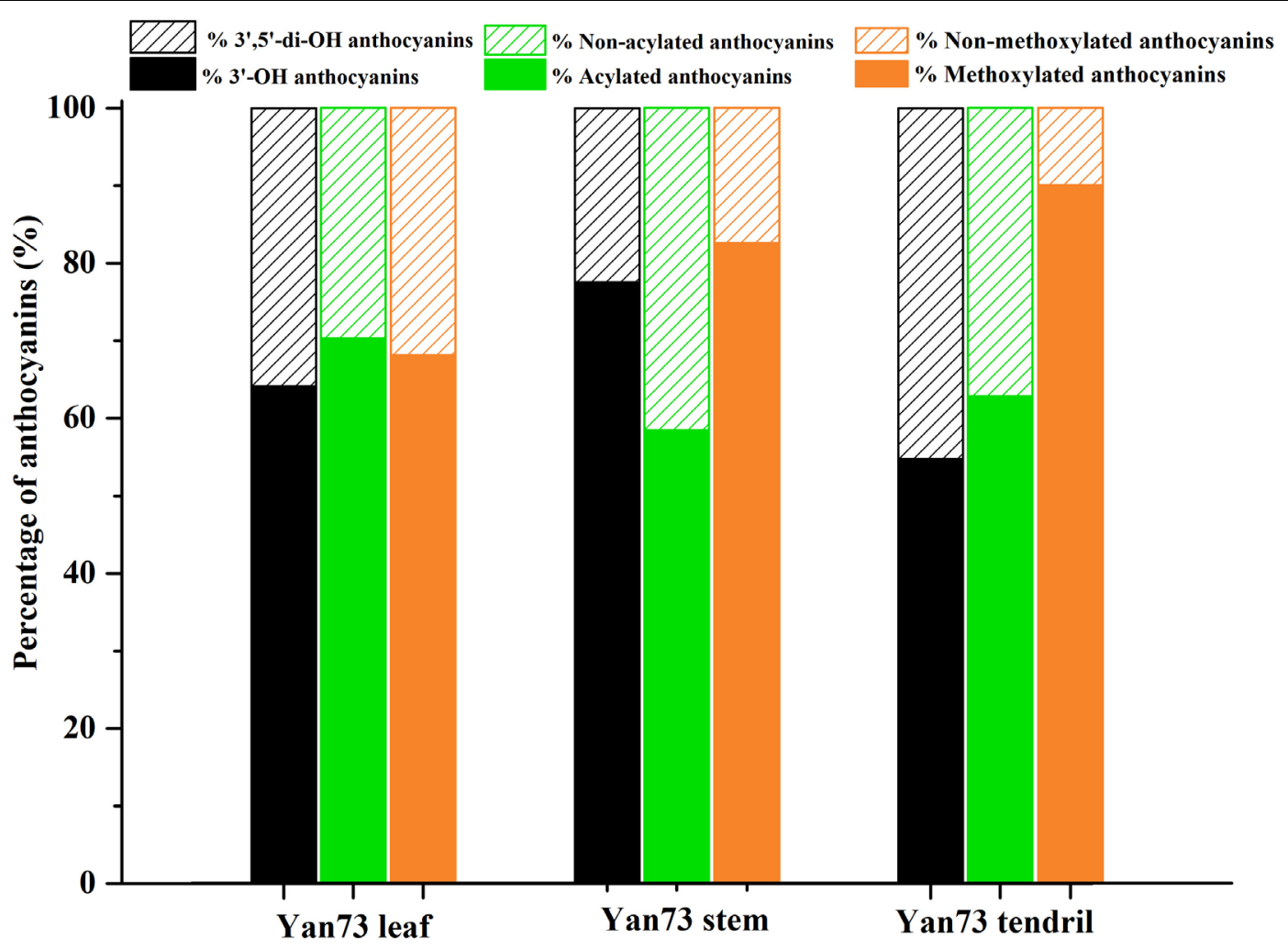

FIGURE 2 | Proportions of different kinds of anthocyanins in Yan73 leaf, stem, and tendril tissues.

tissues (Table 1). These findings were consistent with those of earlier analyses of the Yan73 leaf lamina (Guan et al., 2012). Furthermore, the percentages of 3 '-OH, acylated, and methoxylated anthocyanins in Yan73 leaves, stems, and tendrils were higher than those of the corresponding $3^{\prime}, 5^{\prime}$-di-OH, non-acylated, and non-methoxylated anthocyanins (Figure 2). Similarly, Matus et al. (2017) determined that the Pinot Noir young leaves and tendrils and the Corvina Veronese buds tend to accumulate $3^{\prime}-\mathrm{OH}$ anthocyanins. The similar anthocyanin profiles in the Yan73 leaves, stems, and tendrils indicated that the anthocyanin biosynthesis in these vegetative tissues is regulated by similar mechanisms.

In contrast, no anthocyanins were detected in the Yan73 male parent (Muscat Hamburg) leaves, stems, and tendrils, implying that the anthocyanin accumulation in the Yan73 leaves, stems, and tendrils is attributed to a fully active anthocyanin biosynthesis pathway.

\section{Genes Related to Anthocyanin Biosynthesis in Yan73 Vegetative Tissues}

To explore why the anthocyanin biosynthesis pathway (Figure 3A) is activated in Yan73 leaves, stems and tendrils, the transcriptomes of these three tissues were compared between Yan73 and its male parent. A total of 32 differentially expressed genes (DEGs) related to the anthocyanin biosynthesis pathway were identified in the leaves, whereas 18 and 11
DEGs were detected in the stems and tendrils, respectively (Figure 3B). With the exception of two $V v G S T$ isogenes (NCBI accession number 100258402 and 100251558), other 30 DEGs in leaves exhibited up-regulated expression in Yan73, including early biosynthesis genes [VvPAL(100233012, 100241377 and 100241575), $\quad V v C 4 H(100253493), \quad V v 4 C L(100245991$ and 100254698), $V v C H S(100232843,100263443$, and 100258106), $V v C H I\left(100233078\right.$ and 100255217), $V v F 3^{\prime} H(100232999)$, $V v F 3^{\prime} 5^{\prime} H(100232896), \quad$ and $V v F 3 H(100233079$ and 100253950)], late biosynthesis genes $[\operatorname{VvDFR}(100233141)$ and $\operatorname{VvLDOX}(100233142)]$, anthocyanin modification genes [VvUFGT(100233099, 100247914, and 100247997), $\operatorname{VvOMT(100250579)\quad (Hugueney~et~al.,~2009),~and~}$ $\operatorname{Vv3AT}(100249426,100259716,100261365$, and 100263140) (Rinaldo et al., 2015)], and an anthocyanin transport gene [VvGST4(100232976) (Conn et al., 2008)] (Figure 3B). Of the DEGs in the stems and tendrils, 17 and 10 were more highly expressed in Yan73 than in Yan73 male parent, respectively. Specifically, three types of anthocyanin modification genes ( $V v U F G T, V v O M T$, and $V v 3 A T$ ), and an anthocyanin transport gene ( $V v G S T 4$ ) exhibited up-regulated expression in Yan73 stems and tendrils (Figure 3B). However, $V v C 4 H$ (100253493) and VvDFR (100233141) expression levels were down-regulated in Yan73 stems and tendrils (Figure 3B), respectively. The F3' $\mathrm{H}$ and F3' 5 ' $\mathrm{H}$ enzymes are important for controlling the production of $3^{\prime}-\mathrm{OH}$ anthocyanins and $3^{\prime}, 5^{\prime}-\mathrm{di}-\mathrm{OH}$ anthocyanins in anthocyanin biosynthetic pathway (Figure 3A). In this study, 

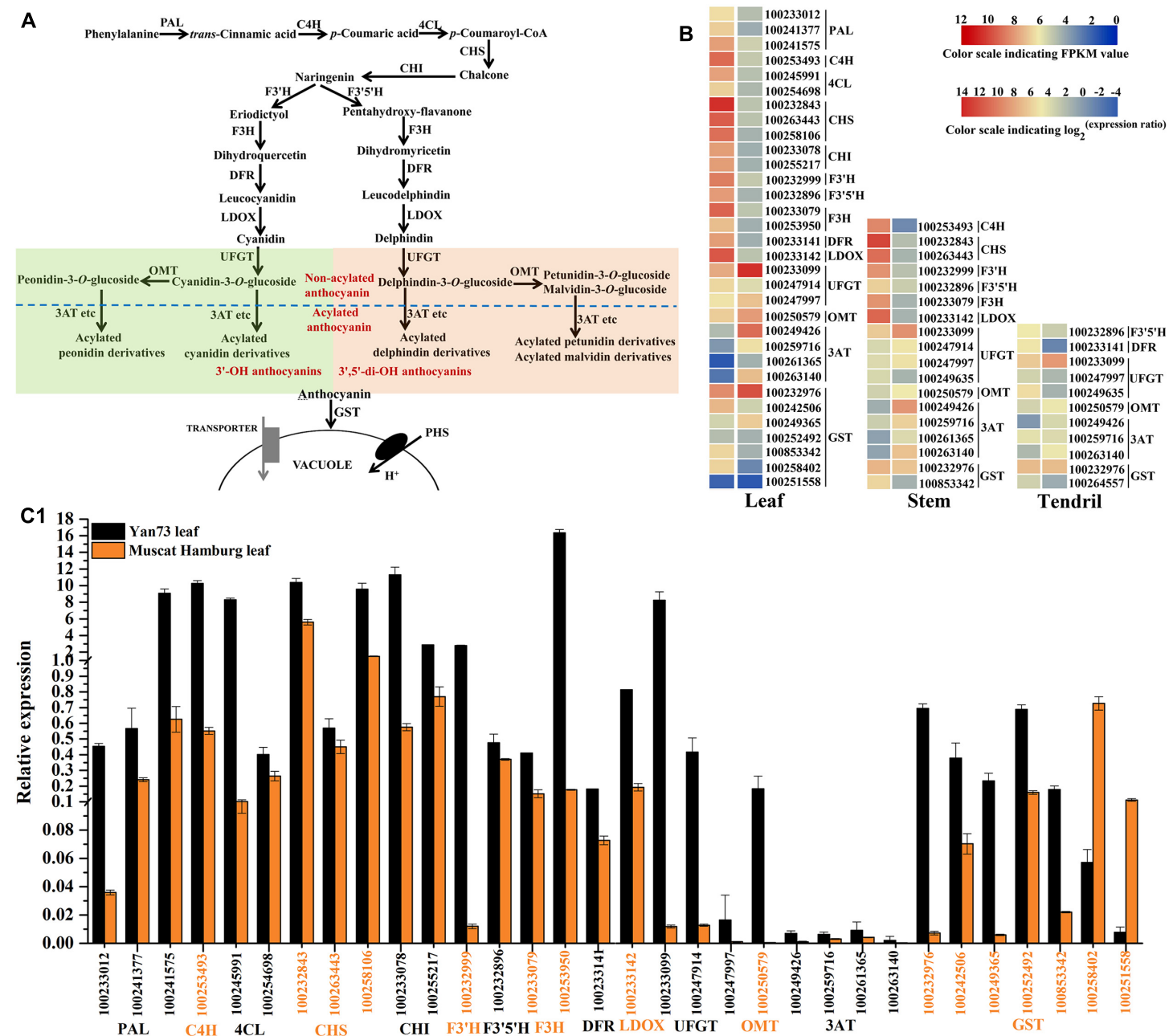

Gene

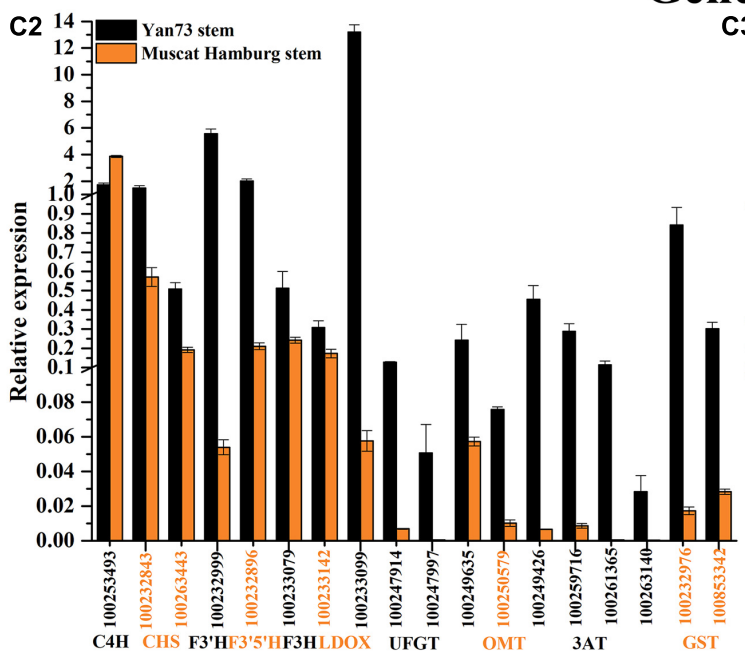

Gene

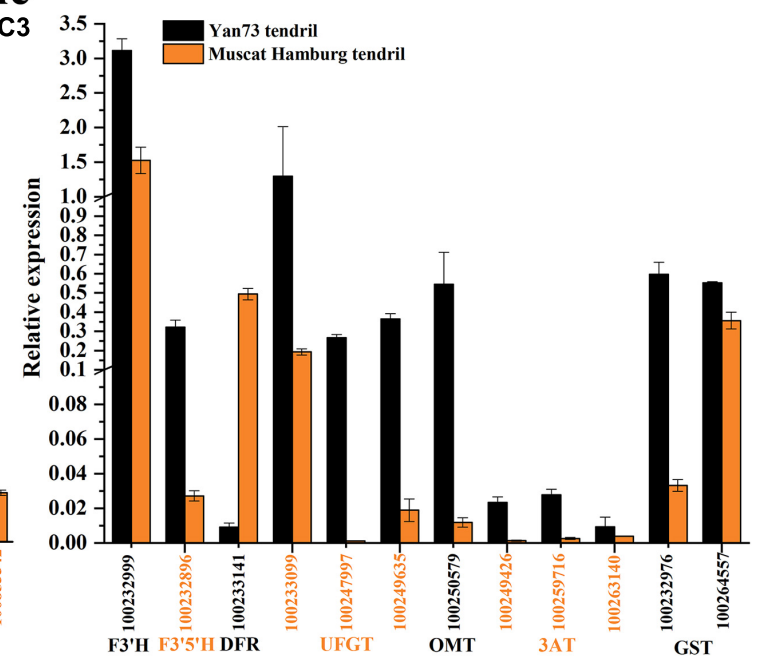

Gene

FIGURE 3 | Expression analysis of anthocyanin biosynthesis genes in Yan73 and its male parent (Muscat Hamburg) leaf, stem and tendril tissues. (A) Schematic of anthocyanin biosynthesis pathways in grapevine vegetative tissues. PAL, phenylalanine ammonia lyase; $\mathrm{C} 4 \mathrm{H}$, trans-cinnamate 4-monooxygenase; 4CL, 4-coumarate: CoA ligase; $\mathrm{CHS}$, chalcone synthase; $\mathrm{CHI}$, chalcone isomerase; $\mathrm{F} 3^{\prime} \mathrm{H}$, flavonoid $3^{\prime}$-hydroxylase; F3' $5^{\prime} \mathrm{H}$, flavonoid $3^{\prime}, 5^{\prime}$-hydroxylase; $\mathrm{F} 3 \mathrm{H}$, flavanone 3-hydroxylase; DFR, dihydroflavonol 4-reductase; LDOX, leucoanthocyanidin dioxygenase; UFGT, anthocyanidin 3-O-glucosyltransferase; OMT, 
FIGURE 3 | Continued

O-methyltransferase; 3AT, anthocyanin 3-O-glucoside-6"-O-acyltransferase; GST, glutathione S-transferase. (B) RNA-seq analysis of anthocyanin biosynthesis genes in Yan73 and its male parent leaf, stem, and tendril tissues. Each gene expression pattern is presented on two grids: the left presents the FPKM values of Yan73 red leaf, stem and tendril tissues, whereas the right presents the $\log _{2}($ Yan73leaf,stem,tendril/Yan73 male parent leaf,stem,tendril) values. The grid in the above with six colors indicates the absolute expression levels in Yan73 leaf, stem and tendril, with the FPKM values $0-2^{2}, 2^{2}-2^{4}, 2^{4}-2^{6}, 2^{6}-2^{8}, 2^{8}-2^{10}$, and $2^{10}-2^{12}$ represented by $0-2,2-4,4-6,6-8,8-10$, and 10-12, respectively. (C) qRT-PCR analysis of anthocyanin biosynthesis genes in Yan73 and its male parent leaves (C1), stems (C2), and tendrils (C3). The data were normalized against VVUbiquitin expression data. Error bars illustrate the standard deviations for three biological replicates.

$V v F 3^{\prime} H$ and $V v F 3^{\prime} 5^{\prime} H$ expression levels were up-regulated in Yan73 leaves and stems (Figure 3B), whereas in tendrils, $V v F 3^{\prime} H$ expression levels were slightly higher in Yan73 (FPKM value of 169.85) than in Yan73 male parent (FPKM value of 116.32), but this difference was not significant. Additionally, $V v F 3^{\prime} H$ was more highly expressed than $V v F 3^{\prime} 5^{\prime} H$ in Yan73 leaves, stems, and tendrils (Figure 3B). These findings are confirmed by our qRT-PCR data (Figure 3C).

Consequently, the expression levels of 30,17 , and 10 anthocyanin biosynthesis genes were up-regulated in Yan73 leaves, stems, and tendrils, respectively, indicating anthocyanin biosynthesis in Yan73 vegetative tissues is regulated by transcription factors.

\section{R2R3-MYB Family Genes Responsible for Anthocyanin Biosynthesis in Grapevine Vegetative Tissues}

To identify the key regulators inducing the expression of a set of anthocyanin genes in Yan73 leaves, stems, and tendrils, we screened our RNA-seq data for R2R3-MYB family members. A total of 65, 69, and 50 DEGs encoding R2R3MYB proteins were identified in the leaves (Supplementary Table S2), stems (Supplementary Table S3), and tendrils (Supplementary Table S4), respectively, of which the expression levels of 47, 31, and 31 genes were up-regulated in Yan73 leaves, stems, and tendrils, respectively. Phylogenetic trees comprising these differentially expressed R2R3-MYB genes in the leaves (Supplementary Figure S1), stems (Supplementary Figure S2), and tendrils (Supplementary Figure S3) as well as the genes encoding known MYB anthocyanin regulators from other species were constructed to identify candidate R2R3-MYB transcription factors putatively involved in the anthocyanin biosynthesis in Yan73 leaves, stems, and tendrils. Structural homology among the MYB proteins from various plant species may indicate the pathways they regulate are generally similar, as are their effects (activation or repression) on the pathways (Aharoni et al., 2001). In our phylogenetic trees, anthocyanin-related MYB transcription factors from various plant species were clustered in the same anthocyanin clade (Figure 4), implying these MYBs might have similar functions in regulating anthocyanin biosynthesis. On the basis of our RNA-seq results for grapevine leaves, stems, and tendrils, two groups of R2R3-MYB genes (Subclades I and II) were identified in the anthocyanin clade. Furthermore, the expression levels of $V v M Y B A 1(100233098)$ in Subclade I and VvMYBA5(100248383), VvMYBA6(100243253), and $V v M Y B A 7(100265568)$ in Subclade II were up-regulated in Yan73 leaves, stems, and tendrils, suggesting both groups of genes have a role in the anthocyanin accumulation of these Yan73 vegetative tissues. Among both groups of $V v M Y B A$ genes, $V v M Y B A 5$ is the most highly expressed in Yan73 leaves, stems, and tendrils, with FPKM values of 63.15, 13.446, and 12.52, respectively, followed by VvMYBA6 (Supplementary Tables S2-S4), suggesting VvMYBA5 and VvMYBA6 maybe the predominant regulators of vegetative anthocyanins in Yan73. We did not detect VvMYBA3(100853472) expression in Yan73 leaves, but it was up-regulated in Yan73 stems and tendrils. These results are confirmed by our qRT-PCR data (Figure 5).

The expression of anthocyanin-related genes is regulated not only by transcriptional activators but also by repressors (Aharoni et al., 2001). In grapevine, Cavallini et al. (2015) reported two groups of R2R3-MYB C2 repressors that distinctly regulate different branches of the phenylpropanoid pathway. Specifically, Subgroup I genes repress the biosynthesis of phenolic compounds, whereas Subgroup II genes negatively regulate anthocyanin and proanthocyanidin biosynthesis. In this study, the expression levels of the Subgroup II genes were up-regulated in Yan73 red leaves, stems, and tendrils, especially VvMYBC2L1(100233136) and VvMYBC2-L3(100251783) (Figure 4). These results indicate this group of R2R3-MYB C2 repressors may play a role in anthocyanin accumulation of Yan73 leaf, stem, and tendril. However, the expression of $V v M Y B 4 A(100233133)$ from Subgroup I was down-regulated in Yan73 leaves, although the expression of $V v M Y B 4 B(100245558)$ from Subgroup I was upregulated in Yan73 leaves, stems, and tendrils. These results are confirmed by our qRT-PCR data (Figure 5).

\section{DISCUSSION}

\section{Anthocyanin Profiles Differ Among Yan73 Vegetative and Reproductive Tissues}

Yan73 grapevine leaf, stem and tendril showed similar anthocyanin profile, indicating that anthocyanin biosynthesis in Yan73 vegetative tissues is regulated by similar mechanisms. However, the anthocyanin profiles of the three analyzed Yan73 vegetative tissues differed from those of Yan73 reproductive tissues, such as the berry skin and flesh. Malvidin-3-O-glucoside and peonidin-3-O-glucoside are reportedly the most abundant anthocyanins in Yan73 berry skin and flesh, respectively (Guan et al., 2012), whereas the most abundant anthocyanin in Yan73 leaves, stems, and tendrils is peonidin-3-O-(trans-6-coumaryl)glucoside (Table 1). Furthermore, we previously revealed that $3^{\prime}, 5^{\prime}$-di-OH and non-acylated anthocyanins are the predominant anthocyanins in Yan73 berry skin and that Yan73 berry flesh has 

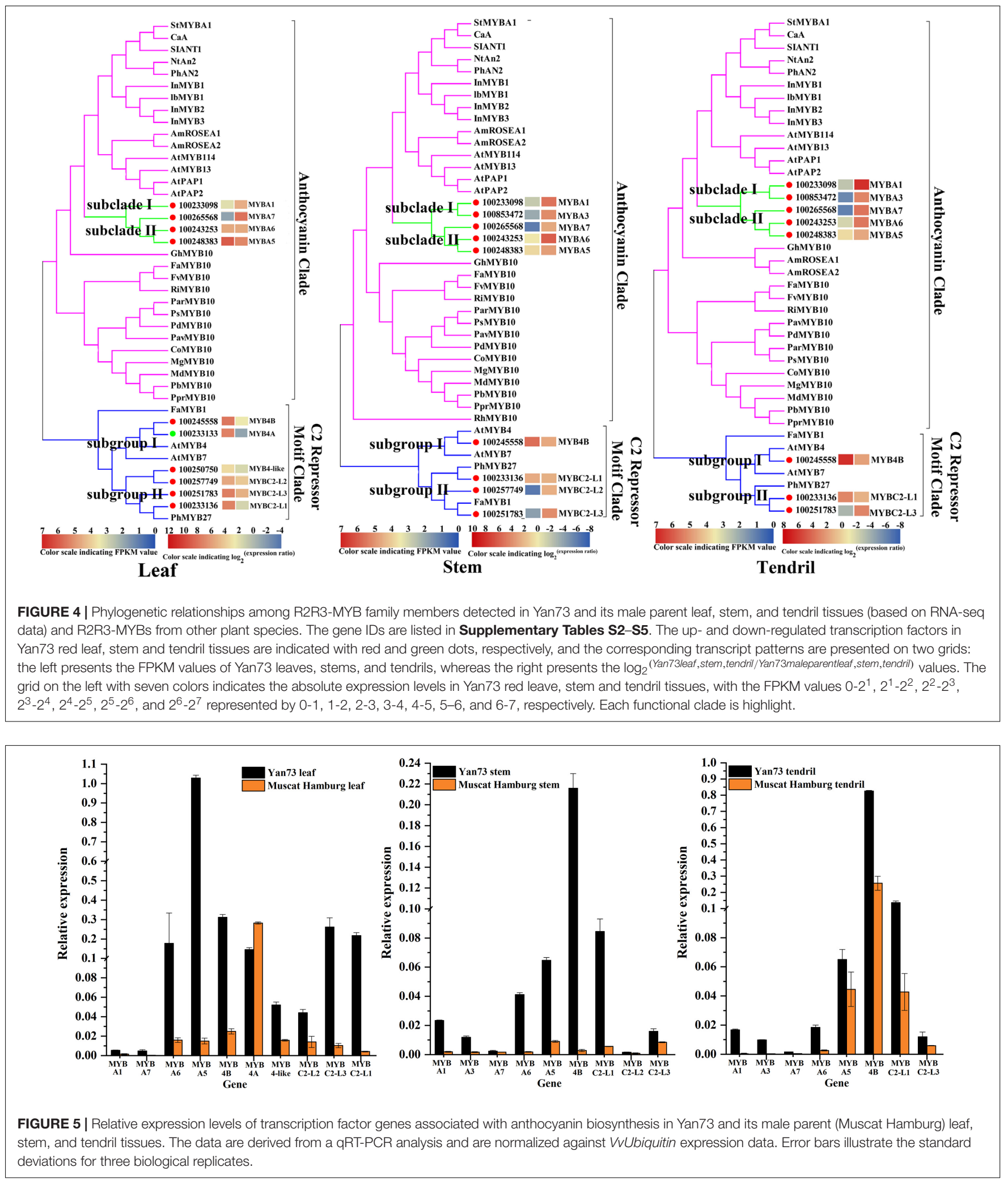

similar concentrations of $3^{\prime}-\mathrm{OH}$ and $3^{\prime}, 5^{\prime}$-di-OH anthocyanins and higher concentrations of non-acylated anthocyanins (Xie et al., 2015, 2018), whereas Yan73 vegetative tissues had higher percentages of $3^{\prime}-\mathrm{OH}$ and acylated anthocyanins. These differences in the anthocyanin profiles of Yan73 vegetative and reproductive tissues suggest that the regulatory mechanisms 
underlying the anthocyanin biosynthesis in these vegetative tissues may differ.

In contrast, no anthocyanins were detected in the vegetative tissues of Yan73 male parent, suggesting anthocyanin biosynthesis in the Yan73 vegetative tissues is attributed to a fully active anthocyanin biosynthesis pathway.

\section{Anthocyanin Biosynthesis Genes Are Activated in Yan73 Vegetative Tissues}

A total of 32 DEGs related to the anthocyanin biosynthesis pathway were identified in the leaves, whereas 18 and 11 DEGs were detected in the stems and tendrils, respectively (Figure 3B). This was consistent with the observed greater number of anthocyanins and higher anthocyanin concentration in Yan73 leaves than in the stems and tendrils (Table 1). Among these DEGs, the expression levels of 30,17, and 10 anthocyanin biosynthesis genes were up-regulated in Yan73 leaves, stems, and tendrils, respectively (Figure 3B). Only Two $V v G S T$ isogenes, $V v C 4 H$ and $V v D F R$ expression levels were down-regulated in Yan73 leaves, stems and tendrils (Figure 3B), respectively, indicating slight differences in the activities of anthocyanin pathways among Yan73 these tissues. In fact, $C 4 H$ and $D F R$ genes do not specifically regulate anthocyanin biosynthesis, but they are also involved in the production of other phenylpropanoid compounds, such as flavanols, flavonols (Xie et al., 2019), hydroxycinnamic acids (Cavallini et al., 2015), and stilbenes (Holl et al., 2013).

The F3' $\mathrm{H}$ and $\mathrm{F} 3{ }^{\prime} 5^{\prime} \mathrm{H}$ enzymes are important for controlling the production of $3^{\prime}-\mathrm{OH}$ anthocyanins and $3^{\prime}, 5^{\prime}-\mathrm{di}-\mathrm{OH}$ anthocyanins (Figure 3A). In this study, $V v F 3^{\prime} H$ was more highly expressed than $V v F 3^{\prime} 5^{\prime} H$ in Yan73 vegetative tissues (Figure 3B), which is consistent with the observed higher $3^{\prime}-\mathrm{OH}$ anthocyanin concentration than $3^{\prime}, 5^{\prime}$-di-OH anthocyanin concentration in Yan73 vegetative tissues. The final anthocyanin modification steps in the grapevine anthocyanin biosynthesis pathway include the glycosylation by UFGT, methylation by OMT, and acylation by $3 \mathrm{AT}$ (Figure $\mathbf{3 A}$ ). In this study, the expression levels of $V v U F G T, V v O M T$, and $V v 3 A T$ were upregulated in the Yan73 vegetative tissues (Figure 3B), which explains the higher percentages of acylated and methoxylated anthocyanins than those of the corresponding non-acylated, and non-methoxylated anthocyanins in Yan73 these tissues (Figure 2). Earlier investigations revealed that VvUFGT (Kobayashi et al., 2001), VvOMT (Hugueney et al., 2009), and Vv3AT (Conn et al., 2008) are critical for anthocyanin biosynthesis in grape berry. In our study, these three types of genes are also important for the anthocyanin biosynthesis in Yan73 vegetative tissues. Of five grapevine GST genes analyzed by Conn et al. (2008), only VvGST1 and VvGST4 are involved in anthocyanin transport. In the current study, we did not detect $V v G S T 1$ expression, but the VvGST4 (100232976) expression was strongly up-regulated in Yan73 vegetative tissues (Figure 3B), suggesting VvGST4 gene may control the anthocyanin transport of Yan73 these vegetative tissues. To the best of our knowledge, there has been relatively little research on the anthocyanin biosynthesis in the teinturier grapevine vegetative tissues.
Only Jeong et al. (2006) reported that VvUFGT expression was detected in the vegetative tissues of a teinturier grapevine cultivar (Bailey Alicant A) and might be responsible for anthocyanin biosynthesis of Bailey Alicant A vegetative tissues.

Consequently, the expression levels of 30, 17, and 10 anthocyanin biosynthesis genes were up-regulated in Yan73 leaves, stems, and tendrils, respectively. Especially, the anthocyanin-specific genes $V v U F G T, V v O M T, V v 3 A T$, and $V v G S T 4$ exhibited up-regulated expression in these Yan73 vegetative tissues. These results imply that anthocyanin biosynthesis in Yan73 vegetative tissues is regulated by transcription factors. Transcription factors, as different from most proteins encoded by structural genes, tend to control multiple pathway steps. Moreover, transcription factor families, including MYB, bHLH, WD40, WRKY, and NAC have been reported to coordinately regulate the expression of a series of anthocyanin biosynthesis genes to control anthocyanin production (Zhou et al., 2015; Amato et al., 2016; Xie et al., 2019).

\section{Transcriptional Activators and Repressors Coordinately Regulate Anthocyanin Biosynthesis in Grapevine Vegetative Tissues}

Recently, extensive research has confirmed that the expression of anthocyanin biosynthesis genes is controlled by the MBW protein complex, with R2R3-MYB transcription factors playing a central role in the coordinated activation of genes specific to anthocyanin pathways (Albert et al., 2014; Costantini et al., 2015). The expression levels of $V v M Y B A 1$ in Subclade I and $V v M Y B A 5$, $V v M Y B A 6$, and $V v M Y B A 7$ in Subclade II were up-regulated in Yan73 vegetative tissues (Figure 4), suggesting both groups of genes have a role in the anthocyanin accumulation of these Yan73 vegetative tissues. Earlier investigations demonstrated that $M Y B A 1$ and MYBA2 on chromosome 2 determined anthocyanin biosynthesis in grape berry (Walker et al., 2007), whereas MYBA5, MYBA6, and MYBA7 on chromosome 14 control the anthocyanin biosynthesis in grapevine vegetative tissues (Matus et al., 2017). However, Matus et al. (2017) claimed that although MYBA5, MYBA6, and MYBA7 regulate anthocyanin accumulation of grapevine vegetative tissues, it is possible that in particular cases, the anthocyanin biosynthesis of the grapevine vegetative tissues may also be mediated by MYBA1. This possibility is supported by the fact MYBA1 is also expressed in some vegetative tissues able to accumulate anthocyanins, such as the leaves of a teinturier cultivar (Jeong et al., 2006), GLRaV-infected leaves (Gutha et al., 2010), and senescing leaves (Matus et al., 2017). Our study reveals that VvMYBA1 along with $V v M Y B A 5, V v M Y B A 6$, and $V v M Y B A 7$ coordinately regulate anthocyanin biosynthesis in Yan73 vegetative tissues. $V v M Y B A 5, V v M Y B A 6$, and $V v M Y B A 7$ are reportedly insufficient for promoting the accumulation of $3^{\prime}, 5^{\prime}$-di-OH anthocyanins because they are incapable of activating the expression of $F 3^{\prime} 5^{\prime} \mathrm{H}$ genes. However, MYBA1 can induce $F 3^{\prime} 5^{\prime} H$ expression and the corresponding accumulation of $3^{\prime}, 5^{\prime}$-di-OH anthocyanins 
(Matus et al., 2017). Our metabolic data revealed that $3^{\prime}, 5^{\prime}$ di-OH anthocyanins are highly abundant in Yan73 vegetative tissues, although not as abundant as $3^{\prime}-\mathrm{OH}$ anthocyanins (Figure 2). This further support that VvMYBA1 participate in the regulation of anthocyanin biosynthesis in Yan73 vegetative tissues. Additionally, among both groups of $V v M Y B A$ genes, $V v M Y B A 5$ is the most highly expressed in Yan73 vegetative tissues, followed by $V v M Y B A 6$, indicating VvMYBA5 and VvMYBA6 maybe the predominant regulators of vegetative anthocyanins in Yan73. This further explain the relatively high $3^{\prime}-\mathrm{OH}$ anthocyanin concentrations in the Yan73 vegetative tissues (Figure 2). VvMYBA3 expression was not detected in Yan73 leaves, but it was up-regulated in Yan73 stems and tendrils (Figure 4). The VvMYBA3 was previously reported to be non-functional in berry anthocyanin accumulation, likely because of the steric competition between functional VvMYBA1 and VvMYBA2 (Fournier-Level et al., 2009). In our study, the $V v M Y B A 3$ expression level was lower than that of $V v M Y B A 1$ in Yan73 stems and tendrils, implying that $V v M Y B A 3$ may be less involved in anthocyanin biosynthesis of Yan73 vegetative tissue.

Anthocyanin-related gene expression is regulated by transcriptional activators as well as repressors (Aharoni et al., 2001). Previous studies identified many R2R3-MYB transcriptional repressors, including PpMYB18 in peach (Zhou et al., 2019), FaMYB1 and FcMYB1 in strawberry (Aharoni et al., 2001; Salvatierra et al., 2013), PtMYB182 in poplar (Yoshida et al., 2015), and PhMYB27 in petunia (Albert et al., 2011), that function coordinately with transcriptional activators to fine-tune anthocyanin biosynthesis during plant development. In this study, the expression levels of the Subgroup II genes were upregulated in Yan73 vegetative tissues, especially VvMYBC2-L1 and VvMYBC2-L3 (Figure 4), indicating this group of R2R3MYB C2 repressors may play a role in anthocyanin accumulation of Yan73 vegetative tissues. Although Subgroup II genes were identified as transcriptional repressors, they are highly expressed during the active stages of anthocyanin accumulation in Yan73 vegetative tissues. In fact, until now, considering the relationship between the expression of R2R3-MYB repressor genes and anthocyanin accumulation, two expression pattern types have been proposed (Chen et al., 2019). The expression of one class of R2R3-MYB repressor genes, including apple MdMYB16 (Xu et al., 2017), poplar MYB182(Yoshida et al., 2015), and Ginkgo biloba GbMYBF2 (Xu et al., 2014), is negatively associated with anthocyanin accumulation. The expression of these genes prevents the ectopic accumulation of anthocyanins. The other class of repressor genes, including strawberry FaMYB1 (Aharoni et al., 2001), peach PpMYB18 (Zhou et al., 2019), and petunia MYB27 (Albert et al., 2014), are highly expressed during the active stages of anthocyanin accumulation to provide feedback repression to finely control and limit anthocyanin levels. In the current study, the R2R3-MYB C2 repressor genes of Subgroup II belong to the second gene class and are highly expressed during the active stages of anthocyanin accumulation in Yan73 vegetative tissues. To explain this expression pattern, the following negative feedback loop regulating anthocyanin biosynthesis was proposed: activators activate repressors, repressors repress activators, and repressors repress repressors (Albert et al., 2014; Zhou et al., 2019). In this study, the upregulated expression of two groups of VvMYBA transcription activator genes induced anthocyanin accumulation and may have also activated the expression of a set of R2R3-MYB C2 repressor genes to maintain suitable anthocyanin accumulation in Yan73 vegetative tissues. However, the expression pattern behaviors of Subgroup I genes in Yan73 leaves, stems, and tendrils were not consistent. The expression of $V v M Y B 4 B$ from Subgroup I was up-regulated in Yan73 leaves, stems, and tendrils, whereas the expression of $V v M Y B 4 A$ from Subgroup I was down-regulated in Yan73 leaves. Therefore, Subgroup I genes were not well related to anthocyanin accumulation in Yan73 vegetative tissues. In fact, to date, only Subgroup II genes, such as MYB4-like (Perez-Diaz et al., 2016), MYBC2-L1 (Cavallini et al., 2015), MYBC2-L2 (Zhu et al., 2019), and MYBC2-L3 (Munoz et al., 2019), have been confirmed to participate in anthocyanin biosynthesis.

Our transcriptomic and metabolic data indicated that the upregulated expression of two groups of VvMYBA transcription activator genes, including $V v M Y B A 1$ on chromosome 2 and $V v M Y B A 5, V v M Y B A 6$, and $V v M Y B A 7$ on chromosome 14, are responsible for the specific anthocyanin accumulation pattern in Yan73 vegetative tissues, although VvMYBA5 and VvMYBA6 maybe the predominant regulator of vegetative anthocyanins in Yan73. Simultaneously, the expression of a set of R2R3MYB C2 repressor genes, mainly including $V v M Y B C 2-L 1$ and $V v M Y B C 2-L 3$, is activated and involved in the negative regulation of anthocyanin biosynthesis in Yan73 vegetative tissues, thereby maintaining appropriate anthocyanin contents. However, future studies will need to determine why these R2R3-MYB transcriptional regulators are activated in Yan73 vegetative tissues.

\section{CONCLUSION}

Yan73 grapevine leaf, stem and tendril showed similar anthocyanin profile, with relatively high percentages of $3^{\prime}$ $\mathrm{OH}$, acylated and methoxylated anthocyanins in these Yan73 red vegetative tissues. Furthermore, peonidin-3-O-(trans-6coumaryl)-glucoside was the most abundant anthocyanin in Yan73 red vegetative tissues. Our RNA-seq data revealed that a total of 30,17 and 10 anthocyanin biosynthesis genes exhibited up-regulated expression in Yan73 leaf, stem and tendril, respectively. Especially, the expression of anthocyaninspecific genes ( $V v U F G T, V v O M T, V v 3 A T$, and $V v G S T 4)$ were all up-regulated in Yan73 vegetative tissues. These results indicate that anthocyanin biosynthesis in Yan73 vegetative tissues is regulated by transcription factors. Our transcriptomic and metabolic data revealed that the up-regulated expression of two groups of VvMYBA transcription activator genes, including $V v M Y B A 1$ on chromosome 2 and VvMYBA5, VvMYBA6, and $V v M Y B A 7$ on chromosome 14, are responsible for the specific anthocyanin patterns of Yan73 vegetative tissues, with $V v M Y B A 5$ and $V v M Y B A 6$ likely encoding the major regulators of the anthocyanin biosynthesis in Yan73 vegetative tissues. Simultaneously, the expression of a set of R2R3-MYB C2 
repressor genes, mainly including $V v M Y B C 2-L 1$ and $V v M Y B C 2-$ $L 3$, is activated and may negatively regulate anthocyanin biosynthesis in Yan73 vegetative tissues, thereby maintaining suitable anthocyanin levels. The data presented herein contribute to our understanding of anthocyanin biosynthesis in grapevine.

\section{DATA AVAILABILITY STATEMENT}

The datasets generated for this study can be found in the NCBI SRA accession PRJNA610705.

\section{AUTHOR CONTRIBUTIONS}

SX designed the experiments, performed most of experiments, analyzed the data, and wrote the manuscript. $\mathrm{ZZ}$ provided all of the financial support and critical intellectual input into the study design. YL, HWC, JL, and HZC assisted in experiments and discussed the results.

\section{REFERENCES}

Aharoni, A., De Vos, C. H. R., Wein, M., Sun, Z. K., Greco, R., Kroon, A., et al. (2001). The strawberry FaMYB1 transcription factor suppresses anthocyanin and flavonol accumulation in transgenic tobacco. Plant J. 28, 319-332. doi: 10.1046/j.1365-313X.2001.01154.x

Albert, N. W., Davies, K. M., Lewis, D. H., Zhang, H., Montefiori, M., Brendolise, C., et al. (2014). A conserved network of transcriptional activators and repressors regulates anthocyanin pigmentation in eudicots. Plant Cell. 26, 962-980. doi: 10.1105/tpc.113.122069

Albert, N. W., Griffiths, A. G., Cousins, G. R., Verry, I. M., and Williams, W. M. (2015). Anthocyanin leaf markings are regulated by a family of R2R3-MYB genes in the genus Trifolium. New Phytol. 205, 882-893. doi: 10.1111/nph. 13100

Albert, N. W., Lewis, D. H., Zhang, H., Schwinn, K. E., Jameson, P. E., and Davies, K. M. (2011). Members of an R2R3-MYB transcription factor family in Petunia are developmentally and environmentally regulated to control complex floral and vegetative pigmentation patterning. Plant J. 65, 771-784. doi: 10.1111/j. 1365-313X.2010.04465.x

Amato, A., Cavallini, E., Zenoni, S., Finezzo, L., Begheldo, M., Ruperti, B., et al. (2016). A grapevine TTG2-like WRKY transcription factor is involved in regulating vacuolar transport and flavonoid biosynthesis. Front Plant Sci. 7:1979. doi: 10.3389/fpls.2016.01979

Blanco-Ulate, B., Amrine, K. C., Collins, T. S., Rivero, R. M., Vicente, A. R., Morales-Cruz, A., et al. (2015). Developmental and metabolic plasticity of white-skinned grape berries in response to botrytis cinerea during noble rot. Plant Physiol. 169, 2422-2443. doi: 10.1104/pp.15.00852

Butelli, E., Titta, L., Giorgio, M., Mock, H. P., Matros, A., Peterek, S., et al. (2008). Enrichment of tomato fruit with health-promoting anthocyanins by expression of select transcription factors. Nat. Biotechnol. 26, 1301-1308. doi: 10.1038/nbt. 1506

Cavallini, E., Matus, J. T., Finezzo, L., Zenoni, S., Loyola, R., Guzzo, F., et al. (2015). The phenylpropanoid pathway is controlled at different branches by a set of R2R3-MYB C2 repressors in grapevine. Plant Physiol. 167, 1448-1470. doi: $10.1104 /$ pp.114.256172

Chalker-Scott, L. (1999). Environmental significance of anthocyanins in plant stress responses. Photochem. Photobiol. 70, 1-9. doi: 10.1111/j.1751-1097.1999. tb01944.x

Chen, L., Hu, B., Qin, Y., Hu, G., and Zhao, J. (2019). Advance of the negative regulation of anthocyanin biosynthesis by MYB transcription factors. Plant Physiol. Biochem. 136, 178-187. doi: 10.1016/j.plaphy.2019.01.024

Conn, S., Curtin, C., Bezier, A., Franco, C., and Zhang, W. (2008). Purification, molecular cloning, and characterization of glutathione S-transferases (GSTs)

\section{FUNDING}

This work was supported by the China Agriculture Research System for Grape Industry (CARS-29-zp-6).

\section{ACKNOWLEDGMENTS}

We are grateful to the Center for Viticulture and Enology, China Agricultural University, for access to the HPLC-ESIMS/MS equipment.

\section{SUPPLEMENTARY MATERIAL}

The Supplementary Material for this article can be found online at: https://www.frontiersin.org/articles/10.3389/fpls.2020.00527/ full\#supplementary-material

from pigmented Vitis vinifera L. cell suspension cultures as putative anthocyanin transport proteins. J. Exp. Bot. 59, 3621-3634. doi: 10.1093/jxb/ ern217

Costantini, L., Malacarne, G., Lorenzi, S., Troggio, M., Mattivi, F., Moser, C., et al. (2015). New candidate genes for the fine regulation of the colour of grapes. J. Exp. Bot. 66, 4427-4440. doi: 10.1093/jxb/erv159

Falginella, L., Di Gaspero, G., and Castellarin, S. D. (2012). Expression of flavonoid genes in the red grape berry of 'Alicante Bouschet' varies with the histological distribution of anthocyanins and their chemical composition. Planta 236, 1037-1051. doi: 10.1007/s00425-012-1658-2

Fang, J. G., Jogaiah, S., Guan, L., Sun, X., and Abdelrahman, M. (2018). Coloring biology in grape skin: a prospective strategy for molecular farming. Physiol. Plant 164, 429-441. doi: 10.1111/ppl.12822

Fournier-Level, A., Le Cunff, L., Gomez, C., Doligez, A., Ageorges, A., Roux, C., et al. (2009). Quantitative genetic bases of anthocyanin variation in grape (Vitis vinifera L. ssp. sativa) berry: a quantitative trait locus to quantitative trait nucleotide integrated study. Genetics 183, 1127-1139. doi: 10.1534/genetics.109. 103929

Guan, L., Li, J. H., Fan, P. G., Chen, S., Fang, J. B., Li, S. H., et al. (2012). Anthocyanin accumulation in various organs of a teinturier cultivar (Vitis vinifera L.) during the Growing Season. Am. J. Enol. Viticul. 63, 177-184. doi: 10.5344/ajev.2011.11063

Gutha, L. R., Casassa, L. F., Harbertson, J. F., and Naidu, R. A. (2010). Modulation of flavonoid biosynthetic pathway genes and anthocyanins due to virus infection in grapevine (Vitis vinifera L.) leaves. BMC Plant Biol. 10:187. doi: 10.1186/1471-2229-10-187

Holl, J., Vannozzi, A., Czemmel, S., D’Onofrio, C., Walker, A. R., Rausch, T., et al. (2013). The R2R3-MYB transcription factors MYB14 and MYB15 regulate stilbene biosynthesis in Vitis vinifera. Plant Cell. 25, 4135-4149. doi: 10.1105/ tpc.113.117127

Huang, D., Tang, Z., Fu, J., Yuan, Y., Deng, X., and Xu, Q. (2019). CsMYB3 and CsRuby1 form an 'activator-and-repressor' loop for regulation of anthocyanin biosynthesis in citrus. Plant Cell Physiol. 61, 318-330. doi: 10.1093/pcp/pcz198

Hugueney, P., Provenzano, S., Verries, C., Ferrandino, A., Meudec, E., Batelli, G., et al. (2009). A novel cation-dependent O-methyltransferase involved in anthocyanin methylation in grapevine. Plant Physiol. 150, 2057-2070. doi: 10. 1104/pp.109.140376

Jeong, S. T., Goto-Yamamoto, N., Hashizume, K., Kobayashi, S., and Esaka, M. (2006). Expression of VvmybA1 gene and anthocyanin accumulation in various grape organs. Am. J. Enol. Viticul. 57, 507-510. doi: 10.1007/s11103-0089446-x

Kobayashi, S., Ishimaru, M., Ding, C. K., Yakushiji, H., and Goto, N. (2001). Comparison of UDP-glucose : flavonoid 3-O-glucosyltransferase (UFGT) gene 
sequences between white grapes (Vitis vinifera) and their sports with red skin. Plant Sci. 160, 543-550. doi: 10.1016/S0168-9452(00)00425-8

Ma, D., Reichelt, M., Yoshida, K., Gershenzon, J., and Constabel, C. P. (2018). Two R2R3-MYB proteins are broad repressors of flavonoid and phenylpropanoid metabolism in poplar. Plant J. 96, 949-965. doi: 10.1111/tpj.14081

Matus, J. T., Cavallini, E., Loyola, R., Holl, J., Finezzo, L., Dal Santo, S., et al. (2017). A group of grapevine MYBA transcription factors located in chromosome 14 control anthocyanin synthesis in vegetative organs with different specificities compared with the berry color locus. Plant J. 91, 220-236. doi: 10.1111/tpj. 13558

Munoz, C., Fanzone, M., and Lijavetzky, D. (2019). Transcriptional regulation of the anthocyanin biosynthesis pathway in developing grapevine berries in cultivar 'Malbec' by putative R2R3 MYB negative regulators. Sci. Hortic. 257:108663. doi: 10.1016/j.scienta.2019.108663

Perez-Diaz, J. R., Perez-Diaz, J., Madrid-Espinoza, J., Gonzalez-Villanueva, E., Moreno, Y., and Ruiz-Lara, S. (2016). New member of the R2R3MYB transcription factors family in grapevine suppresses the anthocyanin accumulation in the flowers of transgenic tobacco. Plant Mol. Biol. 90, 63-76. doi: 10.1007/s11103-015-0394-y

Rinaldo, A. R., Cavallini, E., Jia, Y., Moss, S. M. A., McDavid, D. A. J., Hooper, L. C., et al. (2015). A grapevine anthocyanin acyltransferase, transcriptionally regulated by VvMYBA, can produce most acylated anthocyanins present in grape skins. Plant Physiol. 169, 1897-1916. doi: 10.1104/pp.15. 01255

Salvatierra, A., Pimentel, P., Moya-Leon, M. A., and Herrera, R. (2013). Increased accumulation of anthocyanins in Fragaria chiloensis fruits by transient suppression of FcMYB1 gene. Phytochemistry 90, 25-36. doi: 10.1016/j. phytochem.2013.02.016

Santiago, J. L., Gonzalez, I., Gago, P., Alonso-Villaverde, V., Boso, S., and Martinez, M. C. (2008). Identification of and relationships among a number of teinturier grapevines that expanded across Europe in the early 20th century. Austral. J. Grape Wine Res. 14, 223-229. doi: 10.1111/j.1755-0238.2008. 00022.x

Sunitha, S., Loyola, R., Alcalde, J. A., Arce-Johnson, P., Matus, J. T., and Rock, C. D. (2019). The role of UV-B light on small RNA activity during grapevine berry development. G3 Genes Genomes Genet. 9, 769-787. doi: 10.1534/g3.118. 200805

Walker, A. R., Lee, E., Bogs, J., McDavid, D. A., Thomas, M. R., and Robinson, S. P. (2007). White grapes arose through the mutation of two similar and adjacent regulatory genes. Plant J. 49, 772-785. doi: 10.1111/j.1365-313X.2006.02997.x

Xie, S., Qiao, X., Chen, H., Nan, H., and Zhang, Z. (2019). Coordinated regulation of grape berry flesh color by transcriptional activators and repressors. J. Agric. Food Chem. 67, 11815-11824. doi: 10.1021/acs.jafc.9b05234

Xie, S., Song, C. Z., Wang, X. J., Liu, M. Y., Zhang, Z. W., and Xi, Z. M. (2015). Tissue-specific expression analysis of anthocyanin biosynthetic genes in white- and red-fleshed grape cultivars. Molecules 20, 22767-22780. doi: 10. 3390/molecules201219883

Xie, S., Zhao, T., Zhang, Z., and Meng, J. (2018). Reduction of dihydrokaempferol by Vitis vinfera dihydroflavonol 4-reductase to produce orange pelargonidintype anthocyanins. J. Agric. Food Chem. 66, 3524-3532. doi: 10.1021/acs.jafc. $7 \mathrm{~b} 05766$

Xu, F., Ning, Y. J., Zhang, W. W., Liao, Y. L., Li, L. L., Cheng, H., et al. (2014). An R2R3-MYB transcription factor as a negative regulator of the flavonoid biosynthesis pathway in Ginkgo biloba. Funct. Integr. Genomics 14, 177-189. doi: 10.1007/s10142-013-0352-1

Xu, H. F., Wang, N., Liu, J. X., Qu, C. Z., Wang, Y. C., Jiang, S. H., et al. (2017). The molecular mechanism underlying anthocyanin metabolism in apple using the MdMYB16 and MdbHLH33 genes. Plant Mol. Biol. 94, 149-165. doi: 10.1007/s11103-017-0601-0

Yang, C., Xie, F., Jiang, Y., Li, Z., Huang, X., and Li, L. (2018). Phytochrome a negatively regulates the shade avoidance response by increasing auxin/indole acidic acid protein stability. Dev. Cell 44, 29.e4-41.e4. doi: 10.1016/j.devcel. 2017.11.017

Yoshida, K., Ma, D., and Constabel, C. P. (2015). The MYB182 protein down-regulates proanthocyanidin and anthocyanin biosynthesis in poplar by repressing both structural and regulatory flavonoid genes. Plant Physiol. 167, 693-710. doi: 10.1104/pp.114.253674

Zhou, H., Lin-Wang, K., Wang, F., Espley, R. V., Ren, F., Zhao, J., et al. (2019). Activator-type R2R3-MYB genes induce a repressor-type R2R3-MYB gene to balance anthocyanin and proanthocyanidin accumulation. New Phytol. 221, 1919-1934. doi: 10.1111/nph.15486

Zhou, H., Lin-Wang, K., Wang, H., Gu, C., Dare, A. P., Espley, R. V., et al. (2015). Molecular genetics of blood-fleshed peach reveals activation of anthocyanin biosynthesis by NAC transcription factors. Plant J. 82, 105-121. doi: 10.1111/ tpj.12792

Zhu, Z., Li, G., Liu, L., Zhang, Q., Han, Z., Chen, X., et al. (2019). A R2R3MYB transcription factor, VvMYBC2L2, functions as a transcriptional repressor of anthocyanin biosynthesis in grapevine (Vitis vinifera L.). Molecules 24:92. doi: 10.3390/molecules24010092

Conflict of Interest: The authors declare that the research was conducted in the absence of any commercial or financial relationships that could be construed as a potential conflict of interest.

Copyright (c) $2020 \mathrm{Xie}$, Lei, Chen, Li, Chen and Zhang. This is an open-access article distributed under the terms of the Creative Commons Attribution License (CC BY). The use, distribution or reproduction in other forums is permitted, provided the original author(s) and the copyright owner(s) are credited and that the original publication in this journal is cited, in accordance with accepted academic practice. No use, distribution or reproduction is permitted which does not comply with these terms. 